\title{
Spectinomycin hydrochloride in the treatment of gonorrhoea: Its effect on associated Chlamydia trachomatis infections
}

\author{
J. D. ORIEL, G. L. RIDGWAY, S. TCHAMOUROFF, AND JEAN OWEN \\ From the Departments of Genito-Urinary Medicine and Clinical Microbiology, \\ University College Hospital, London
}

SUMMARY Sixty-three heterosexual men were successfully treated with a single injection of spectinomycin hydrochloride $2 \mathrm{~g}$ for urethral infections with Neisseria gonorrhoeae. Chlamydia trachomatis was recovered from the urethra of 11 of these men both before and after treatment. In six men, the organism was isolated after but not before treatment. No isolates were obtained from the remaining men either before or after treatment. All 17 of the men who yielded C. trachomatis developed post-gonococcal urethritis. Eight of 46 men from whom no isolate was obtained in their cultures developed post-gonococcal urethritis. Seventeen of 50 women successfully treated with spectinomycin for cervical infections with $N$. gonorrhoeae yielded isolates of $C$. trachomatis both before and after treatment. The organism was isolated from five women before but not after treatment, and from four women after but not before treatment. In 24 women culture for $C$. trachomatis was negative both before and after treatment. Spectinomycin hydrochloride in the dosage used rarely eliminated $C$. trachomatis from the genital tract of either men or women; in this respect it resembled two other drugs commonly used for the treatment of gonorrhoea-penicillin and ampicillin.

\section{Introduction}

Patients with genital infections with Neisseria gonorrhoeae often have a concomitant infection with Chlamydia trachomatis. This organism, which is an important cause of non-gonococcal urethritis (Holmes, et al., 1975; Oriel et al., 1976a), has been isolated from 20 to $30 \%$ of men with gonococcal urethritis (Richmond et al., 1972; Oriel et al., 1976a) and from 30 to $60 \%$ of women with gonococcal cervicitis (Wentworth et al., 1973; Hilton et al., 1974; Oriel et al., 1974). Several antibacterial agents in dosages that are curative for $N$. gonorrhoeae infections are ineffective against coexisting $C$. trachomatis infections. Thus gentamicin completely fails to eliminate $C$. trachomatis (Oriel

Address for reprints: J. D. Oriel, Department of Genito-Urinary Medicine, University College Hospital, London

Received for publication 20 May 1977 et al., 1975), and both penicillin and ampicillin show only weak antichlamydial activity (Richmond et al., 1972; Oriel et al., 1976b). Most men with infection of the urethra by $C$. trachomatis which persists after treatment for gonorrhoea develop post-gonococcal urethritis (PGU) (Richmond et al., 1972; Oriel et al., 1975).

Spectinomycin, an aminocyclitol antibiotic, has given good results in the treatment of gonorrhoea (Cornelius and Domescik, 1970; Schroeter et al., 1975), and has been recommended as the drug of choice for patients not cured by other antimicrobials (Venereal Disease Advisory Committee, 1974). Its clinical effect against $C$. trachomatis thus becomes important, although in vitro studies indicate only marginal activity (Ridgway et al., 1976). We are reporting the effect of a single intramuscular injection of spectinomycin hydrochloride $2 \mathrm{~g}$ on $C$. trachomatis associated with $N$. gonorrhoeae in both men and women. Cell culture for $C$. trachomatis was peformed before and after treatment in all 
cases and the development of PGU in both Chlamydia-positive and Chlamydia-negative men was noted.

\section{Materials and methods}

All patients were seen in the Department of GenitoUrinary Medicine, University College Hospital, London between August 1976 and February 1977, and informed consent was obtained before inclusion in the study. After local and general examination, specimens were collected as described below.

\section{SPECIMEN COLLECTION FROM MEN}

A specimen of urethral secretion was taken from each man with a disposable plastic loop and spread on a slide for Gram staining and microscopical examination. A second specimen was inoculated on to a selective culture medium for $N$. gonorrhoeae. A cotton-wool tipped wire endourethral swab was then inserted 4-5 cm into the urethra. On withdrawal, the swab was transferred to $2 \mathrm{ml}$ of transport medium for $C$. trachomatis. A two-glass urine test was carried out at each visit, and serological tests for syphilis were also performed.

SPECIMEN COLLECTION FROM WOMEN

Women were examined in the lithotomy position. The cervix was exposed with a Cusco speculum. Urethral and cervical specimens for microscopical examination and culture for $N$. gonorrhoeae were taken using plastic disposable loops. A specimen from the posterior vaginal pool was also taken for microscopy and culture for Trichomonas vaginalis. The cervix was then cleaned with cotton-wool, and a cotton-wool tipped swab rotated in the cervical canal. On removal, the swab was transferred to $2 \mathrm{ml}$ of transport medium for culture for $C$. trachomatis. Rectal specimens for culture for $N$. gonorrhoeae were taken from some patients, but cultures for Chlamydia were not performed on rectal specimens. Serological tests for syphilis were included for all patients.

In cases in which gonorrhoea was diagnosed, the patient (male or female) was given $2 \mathrm{~g}$ spectinomycin hydrochloride by intramuscular injection. Each patient was asked to return for re-examination after 3 days, 7 days, and 14 days. There was some individual variation in follow-up attendance, but in many cases it was possible to follow-up patients for longer periods. At each attendance the diagnostic tests described above were repeated. Patients were requested to refrain from sexual intercourse during the follow-up period.
ASSESSMENT OF PGU

PGU was defined as the presence of $\geqslant 20$ polymorphonuclear leucocytes in at least three fields of a Gram-stained urethral smear examined at a magnification of $\times 900$ (Oriel et al., 1975).

\section{LABORATORY METHODS}

$N$. gonorrhoeae was cultured on a selective medium (Phillips et al., 1972). Cell culture for C. trachomatis was by the method of Reeve et al. (1975), using McCoy cells pre-treated with idoxuridine.

\section{Results}

MEN

A group of 114 heterosexual men with gonococcal urethritis was treated. Of these men, 51 were excluded for the following reasons: default (38), initial contaminated Chlamydia culture (8), treatment failure at first follow-up examination (1), reinfection with $N$. gonorrhoeae during follow-up (4). Thus, full results were available for 63 men. Cultures for $C$. trachomatis were positive at the initial visit in $11(17.5 \%)$ of these men. A further six men yielded isolates during follow-up, for an overall isolation rate of $27 \cdot 0 \%$ (Table 1 ).

Table 1 Effect of a single dose of spectinomycin ( $2 \mathrm{~g}$ intramuscularly) on the frequency of isolations of Chlamydia trachomatis and on the development of post-gonoccccal urethritis (PGU) in 63 heterosexual men with gonococcal urethritis

\begin{tabular}{lll}
\hline Isolate of C. trachomats c: : :i:ed & $\begin{array}{l}\text { No. of patients } \\
\text { developing PGU/ } \\
\text { no. with isolation } \\
\text { pattern }\end{array}$ \\
\hline Before treciment & After treatment & $11 / 11$ \\
\hline Yes & Yes & $0 / 0$ \\
Yes & No & $6 / 6$ \\
No & Yes & $8 / 46$ \\
No & No & \\
\hline
\end{tabular}

Cultures for $C$. trachomatis were positive both before and after treatment in 11 patients. The intervals between isolations were one week or less for six men, two weeks for four men, and three weeks for one man. No patient who initially yielded $C$. trachomatis became isolation-negative after treatm:nt Six patients who were initially isolation-regative became isolation-positive after treatment, the intervals being one week for one man, two weeks tor one man, and three weeks for four men. In 46 patients, isolates of $C$. trachomatis were not obtained befice or after treatment; 13 of this group were follow d-up for two weeks, 16 for three weeks, and 17 for iour weeks. 
After treatment with spectinomycin, 25 of the 63 patients with gonococcal urethritis $(39.7 \%)$ developed PGU. All 17 men with positive cultures for C. trachomatis after treatment and eight of 46 men with negative cultures $(17.4 \%)$ developed PGU.

\section{WOMEN}

A series of 55 women with confirmed gonococcal cervicitis was treated, but five were excluded (default (3), reinfection with $N$. gonorrhoeae during follow-up (1), initial contamination (1)). There were no treatment failures. Thus, results were available for 50 women (Table 2). Of these, $22(44 \%)$ yielded $C$. trachomatis from the cervix on first examination and a further four during follow-up for an overall isolation rate of $52 \%$. C. trachomatis was isolated from 17 women both before and after treatment. The intervals between examinations were one week or less for three women, two weeks for six women, three weeks for three women, and four weeks or more for five women. C. trachomatis was isolated from five patients before treatment but not subsequently, the intervals being two weeks (1 woman), three weeks (1 woman), and four weeks ( 3 women). C. trachomatis was not isolated from four patients before treatment but was isolated subsequently, the intervals being one week (1 woman), three weeks (2 women), and over four weeks (1 woman). Finally, C. trachomatis was not isolated from 24 women either before or after treatment, with intervals between examinations of two weeks ( 7 women), three weeks ( 3 women), and over four weeks (14 women).

Table 2 Effect of a single dose of spectinomycin (2 $\mathrm{g}$ intramiscularly) on the frequency of isclation of Chlamydia trachomatis from the cervix of 50 women with gonococcal cervicitis

\begin{tabular}{lll}
\hline \multicolumn{2}{l}{ Isolate of C. trachomatis obtained } & \\
\cline { 1 - 2 } Before treatment & After treatment & No. of patients \\
\hline Yes & Yes & 17 \\
Yes & No & 5 \\
No & Yes & 4 \\
No & No & 24 \\
\hline
\end{tabular}

\section{Discussion}

Spectinomycin in the dosage used was an effective treatment for gonorrhoea in our patients and had a low failure rate. However, this antimicrobial had little effect on associated $C$. trachomatis infection in either men or women; in this respect it resembled penicillin and ampicillin.

The failure of the three agents which are the most widely used for the treatment of gonorrhoea to have a significant effect on associated $C$. trachomatis infection indicates an unsatisfactory situation. C. trachomatis is an important genital pathogen. It undoubtedly causes many infections of nongonococcal urethritis, cervicitis, and neonatal eye disease (Dunlop et al., 1966; Rees et al., 1977); it may be implicated in the pathogenesis of salpingitis (Mardh et al., 1977), epididymitis (Harnisch et al., 1977), and neonatal respiratory disease (Beem and Saxon, 1977). Its treatment appears to be desirable. Between 20 and $60 \%$ of patients with gonorrhoea are infected by $C$. trachomatis, but at present only a proportion of the men (those diagnosed as having PGU) and none of the women are likely to receive effective treatment.

Because of the appearance of $\beta$-lactamase producing strains of $N$. gonorrhoeae, the treatment of gonorrhoea is at present being reappraised. Whether one drug will be found which gives good results in the treatment of both $N$. gonorrhoeae and $C$. trachomatis infections remains to be seen, but the time is ripe for further trials of antimicrobials, single, combined, or perhaps sequential, in the treatment of these serious infections.

We are grateful to Dr E. Joan Stokes for kindly providing laboratory facilities and to Ms Lam Po Tang for technical assistance. This work was supported by the Medical Research Council and by Upjohn Ltd.
References

Beem, M. O., and Saxon, E. M. (1977). Respiratory-tract colonisation and a distinctive pneumonia syndrome in infants infected with Chlamydia trachomatis. New England Journal of Medicine, 296, 306-310.

Cornelius, C. E., and Domescik, G. (1970). Spectinomycin hydrochloride in the treatment of uncomplicated gonorrhoea. British Journal of Venereal Diseases, 46, 212-213.

Dunlop, E. M. C., Harper, I. A., Al-Hussaini, M. K., Garland, J. A., Treharne, J. D., Wright, D. J. M., and Jones, B. R. (1966). Relation of TRIC agent to 'non-specific' genital infection. British Journal of Venereal Diseases, 42, 77-87.

Harnisch, J. P., Berger, R. E., Alexander, E. R., Monda, G., and Holmes, K. K. (1977). Aetiology of acute epididymitis. Lancet, 1 , 819-821.

Hilton, A. L., Richmond, S. J., Milne, J. D., Hindley, F., and Clarke, S. K. R. (1974). Chlamydia $A$ in the female genital tract. British Journal of Venereal Diseases, 50, 1-10.

Holmes, K. K., Handsfield, H. H., Wang, S-P., Wentworth, B. B., Turck, M., Anderson, J. B., and Alexander, E. R. (1975). Etiology of non-gonococcal urethritis. New England Journal of Medicine, 292, 1199-2005.

Mardh, P. A., Ripa, T., Svensson, L., and Westrom, L. (1977) Chlamydia trachomatis infection in patients with acute salpingitis. New England Journal of Medicine, 296, 1377-1379. 
Oriel, J. D., Powis, P. A., Reeve, P., Miller, A., and Nicol, C. S. (1974). Chlamydial infections of the cervix. British Journal of Venereal Diseases, 50, 11-16.

Oriel, J. D., Reeve, P., Thomas, B. J., and Nicol, C. S. (1975). Infection with Chlamydia Group $\mathbf{A}$ in men with urethritis due to Neisseria gonorrhoeae. Journal of Infectious Diseases, 131, 376-382.

Oriel, J. D., Reeve, P., Wright, J. T., and Owen, J. (1976a). Chlamydial infection of the male urethra. British Journal of Venereal Diseases, 52, 46-51.

Oriel, J. D., Ridgway, G. L., Reeve, P., Beckingham, D. C., and Owen, J. (1976b). The lack of effect of ampicillin plus probenecid given for genital infections with Neisseria gonorrhoeae on associated infections with Chlamydia trachomatis. Journal of Infectious Diseasès, 133, 568-571.

Phillips, I., Humphrey, D., Middleton, A., and Nicol, C. S. (1972). Diagnosis of gonorrhoea by culture on a selective medium containing vancomycin, colistin, nystatin and trimethoprim (VCNT). A comparison with Gram-staining and immunofluorescence. British Journal of Venereal Diseases, 48, 287-292.

Rees, E., Tait, I. A., Hobson, D., Byng, R. E., and Johnson, F. W. A (1977). Neonatal conjunctivitis caused by Neisseria gonorrhoeae and Chlamydia trachomatis. British Journal of Venereal Diseases, 53, 173-179.
Reeve, P., Owen, J., and Oriel, J. D. (1975). Laboratory procedures for the isolation of Chlamydia trachomatis from the human genital tract. Journal of Clinical Pathology, 28, 910-914.

Richmond, S. J., Hilton, A. L., and Clarke, S. K. R. (1972). Chlamydial infection. Role of Chlamydia subgroup $\mathbf{A}$ in non-gonococcal and post-gonococcal urethritis. British Journal of Venereal Diseases, 48, 437-444.

Ridgway, G. L., Owen, J. M., and Oriel, J. D. (1976). A method for testing the antibiotic susceptibility of Chlamydia trachomatis in a cell culture system. Journal of Antimicrobial Chemotherapy, 2, 71-76.

Schroeter, A. L., Reynolds, G. H., Holmes, K. K., Pyke, T., and Wiesner, P. J. (1975). Spectinomycin in the treatment of gonorrhea. Journal of the American Venereal Disease Association, 1, 139-145.

Venereal Disease Advisory Committee (1974). Gonorrhoea-CDC recommended treatment schedule. Morbidity and Mortality Weekly Report, 23, 341-342, 347-348.

Wentworth, B. B., Bonin, P., Holmes, K. K., Gutman, L., Wiesner, P., and Alexander, E. R. (1973). Isolation of viruses, bacteria and other organisms from venereal disease clinic patients: methodology and problems associated with multiple isolation. Health Laboratory Science, 10, 75-81. 